\title{
Population prevalence of psychiatric disorders in Chile: 6-month and I-month rates ${ }^{\dagger}$
}

\author{
BENJAMIN VICENTE, ROBERT KOHN, PEDRO RIOSECO, SANDRA SALDIVIA, \\ CHRISTINE BAKER and SILVERIO TORRES
}

\section{Background Few South American studies have examined current prevalence rates of psychiatric disorders.}

\begin{abstract}
Aims To examine prevalence rates in a nationally representative adult population from Chile.
\end{abstract}

Method The Composite International Diagnostic Interview was administered to a stratified random sample of 2978 individuals from four provinces representative of the country's population. Six-month and I-month prevalence rates were estimated. Demographic correlates, comorbidity and service use were examined.

\section{Results Nearly a fifth of the Chilean} population had had a psychiatric disorder during the preceding 6 months. The 6 month and I-month prevalence rates were $19.7 \%$ and $16.7 \%$ respectively. For the 6 month prevalence the five most common disorders were simple phobia, social phobia, agoraphobia, major depressive disorder and alcohol dependence. Less than $30 \%$ of those with any psychiatric diagnosis had a comorbid psychiatric disorder and the majority of them had sought treatment from mental health services.

\section{Conclusions Current prevalence} studies are useful indicators of service needs. People with comorbid psychiatric conditions have high rates of service use. The low rate of comorbidity in Chile merits further study.

Declaration of interest None. Funding detailed in Acknowledgements.

†See editorial, pp. 289-290, this issue.
Mental disorders are a growing public health concern in Latin America, as elsewhere in the world. By the year 2020, the proportion of the contribution of neuropsychiatric conditions to overall disability is expected to be about $20 \%$, compared with only 9\% in 1990 (Murray \& Lopez, 1996). This increasing burden in Latin America may be the result of an epidemiological transition, emergent disorders and changing population structure. In addition, the population of the region is anticipated to increase by $28 \%$ in the next 15 years (World Bank, 2002). Without epidemiological data available to government public health officials there is little support for the 'epidemiological basis for a call to action' made over a decade ago (Levav et al, 1989). The availability of human resources and medications is inadequate to meet even current demands of those with serious mental illness in South America (Larrobla \& Botega, 2001).

The objective of the Chile Psychiatric Prevalence Study (CPPS), based on a nationally representative sample, was to investigate the prevalence and risk factors for mental illness in Spanish-speaking South America. This report focuses on the 6-month and 1-month prevalence rates of disorders, and their association with socio-demographic correlates, comorbidity and service use. One-month prevalence identifies individuals who have an acute episode of a disorder (both incident cases and relapses), as well as those with chronic disorders. Six-month prevalence includes people who have recently recovered from an episode of mental illness or whose condition is subclinical, but are still in need of services.

\section{METHOD}

\section{Sample selection}

The CPPS was based on a householdstratified sample of people defined by the health service system to be adults (aged 15 years and older). The study was designed to represent the whole population of the country. Chile is composed of 51 provinces, grouped into 13 regions, and has a population of approximately 14 million. The study sample was selected from four geographically distinct provinces, chosen as being representative of the distribution of the national population: Santiago, Concepción, Iquique and Cautin.

The capital city, Santiago, accounts for one-third of the nation's population. Concepción is located in the central region of Chile and is its second largest city. Iquique is in the north of the country and is a desert region, with isolated towns. The province of Cautin, in the south, is a sparsely populated rural area. The population of Chile are mainly urban dwellers.

In Chile provinces are subdivided into comunas, then into districts, and finally into blocks, each of which were selected randomly. The number of households available on each block was counted. Using the 1992 national census the number of households required on each block was determined. The households were chosen clockwise, starting with the first one on the northern corner of each block. Subsequent households were selected on the basis of a number obtained by dividing the census estimates into the number of residences on the block. A list of inhabitants aged 15 years and older in descending order by age, with males listed first, was then generated. Using 12 randomly pre-assigned Kish tables (Kish, 1965), one person per household was selected from the list to be interviewed.

The survey was conducted by the University of Concepción Department of Psychiatry between July 1992 and June 1999. The sites were completed in the following order as funding was secured: Concepción, Santiago, Iquique and Cautin. A total of 2987 individuals participated in the survey, with a response rate of $90.3 \%$. Weighting was used to account for the probability of the comuna, district, block, household and respondent being selected. The data were adjusted to the 1992 national census, based on age, gender and marital status, using a second weighting.

\section{Diagnostic assessment}

The structured diagnostic interview schedule used to generate the diagnoses 
was the Composite International Diagnostic Interview (CIDI) versions 1.0 and 1.1 (Robins et al, 1988), conducted by welltrained lay interviewers. The DSM-III-R diagnostic criteria were employed (American Psychiatric Association, 1987). As these versions of the CIDI did not include post-traumatic stress disorder (PTSD) and antisocial personality disorder, the corresponding sections of the Diagnostic Interview Schedule (DIS; Robins et al, 1981) were included in the interview, in all study areas except Cautin. A section on health service use in the 6 months prior to the interview was also included in the interview schedule.

The translation into Spanish was conducted using the protocol outlined by the World Health Organization (WHO; Sartorius \& Kuyken, 1994). The translated CIDI underwent a validation study and was found to have kappa values ranging from 0.52 for somatisation to 0.94 for affective disorders (Vielma et al, 1992). The DIS sections were similarly translated and validated (Rioseco et al, 1992) with $\kappa$ of 0.72 for antisocial personality disorder and 0.63 for PTSD.

Diagnoses were generated after double data entry and verification for logical inconsistencies using the CIDI computer programs for versions 1.0 and 1.1. The DSM-III-R diagnoses included in the CPPS were major depression, mania, dysthymia, panic disorder, agoraphobia, alcohol abuse, alcohol dependence, drug abuse, drug dependence, nicotine dependence, antisocial personality disorder, somatisation disorder and non-affective psychosis. Nonaffective psychosis is a summary category consisting of schizophrenia, schizophreniform disorder and schizoaffective disorder. The CIDI sections for eating disorders, obsessive-compulsive disorder, simple phobia and social phobia were not included in the first two sites and therefore are not represented in the overall rates of anxiety disorders nor in the 'any disorder' category.

Service use, both private and public sector, in the past 6 months was investigated for both specialist and non-specialist mental health services: 'specialist mental health services' were defined as out-patient treatment by a mental health care professional, or psychiatric hospitalisation, with a sub-category of treatment for substance misuse; 'non-specialist mental health services' were defined as psychiatric care delivered by the formal health care system that was not provided by a mental health care professional.

\section{Interviewers and training}

The interviewers were all university students in their senior year studying social sciences. Medical students were excluded in case respondents might misinterpret questions about last seeing a health care professional. Training was conducted following WHO protocol at the University of Concepción (a CIDI training and reference centre), and it consisted of over $80 \mathrm{~h}$ of instruction and practice sessions. Each interviewer had to conduct practice interviews with adult volunteers (with and without psychiatric disorders) selected from local clinics, as well as a pilot interview on an individual in a non-selected household in the community, as part of the training. These interviews were audiotaped and reviewed with the trainers. Of the 163 students originally trained, only $64(39 \%)$ were accepted as interviewers.

Approximately $80 \%$ of the interviews were audiotaped, with the interviewee's consent. About one in five of the audiotapes were randomly reviewed to maintain quality control, in addition to recordings of the first three sessions conducted by each interviewer. Audiotapes were used to correct missing and unclear responses, as well as to confirm the accuracy of the interviews. Interviews were edited according to the guidelines in the CIDI trainers' manual. If edit issues and inconsistencies in the interview could not be clarified, the interviewer was asked to contact the respondents again. In addition, households were randomly selected by the field supervisors for checking to verify that the interview had been conducted in full. This resulted in a number of respondents being interviewed a second time.

\section{Informed consent}

The University of Concepción's institutional review board approved the study. Informed consent was obtained from all respondents. Names of the respondents were not included on the interview schedule, to ensure anonymity during data processing. Respondents were given an opportunity to obtain the results of their CIDI.

\section{Statistical analysis}

The SUDAAN statistical package (Shah et al, 1997), Taylor series linearisation method, was used to estimate the standard errors due to the sample design and the need for weighting. The analysis was conducted using procedures without replacement for non-respondents. The region, province, comuna and district selected were used as the defined strata. Logistic regression with the corresponding $95 \%$ confidence interval was used to examine the association with demographic risk factors. All results, unless otherwise stated, are presented as weighted data.

\section{RESULTS}

\section{Prevalence rate of psychiatric disorders}

Table 1 lists the 6-month and 1-month prevalence rates of the disorders evaluated in the CPPS. The prevalence estimates are presented without exclusion criteria based on the DSM-III-R hierarchy rules. Nearly a fifth $(19.7 \%)$ of the study population had had a psychiatric disorder during the past 6 months. The 1-month prevalence rate was $16.7 \%$. For 6 -month prevalence the five most common disorders were simple phobia $(6.1 \%)$, social phobia $(5.9 \%)$, agoraphobia $(5.1 \%)$, major depressive disorder $(4.7 \%)$ and alcohol dependence $(4.3 \%)$. Alcohol dependence was more common than major depressive disorder for 1-month prevalence. 'Any substance use disorder' was the most prevalent group of psychiatric conditions in the population. If nicotine dependence is excluded, the anxiety disorders, followed by the affective disorders, overtake substance use disorders. Affective disorders as a group were found in $7.9 \%$ in the past 6 months and $6.5 \%$ in the past month.

\section{Socio-demographic correlates of disorders}

Bivariate risk factor associations are reported for broad 6-month diagnostic categories in Table 2, and for 1-month prevalence in Table 3. Affective disorders were twice as common and anxiety disorders over five times as common among women; however, substance use disorders were more than twice as common in men. No increased risk for women was noted in the overall rates.

In comparison with individuals aged 65 years or more, differential risks by age were noted. Most notably, age was not predictive of substance use disorders. For 6month prevalence, those less than 35 years old were at increased risk of affective disorders, whereas those aged 35-64 years 
Table I Six-month and I-month prevalence rates of mental disorders in Chile

\begin{tabular}{|c|c|c|c|c|c|c|c|c|c|c|c|c|}
\hline \multirow[t]{3}{*}{ DSM-III-R diagnosis } & \multicolumn{6}{|c|}{ Six-month prevalence } & \multicolumn{6}{|c|}{ One-month prevalence } \\
\hline & \multicolumn{2}{|c|}{ Male } & \multicolumn{2}{|c|}{ Female } & \multicolumn{2}{|c|}{ Total } & \multicolumn{2}{|c|}{ Male } & \multicolumn{2}{|c|}{ Female } & \multicolumn{2}{|c|}{ Total } \\
\hline & $\%$ & s.e. & $\%$ & s.e. & $\%$ & s.e. & $\%$ & s.e. & $\%$ & s.e. & $\%$ & s.e. \\
\hline \multicolumn{13}{|l|}{ Affective disorders } \\
\hline Major depressive episode & 3.0 & 0.5 & 6.2 & 0.6 & 4.7 & 0.4 & 2.3 & 0.4 & 4.5 & 0.5 & 3.4 & 0.4 \\
\hline Manic episode & 0.7 & 0.3 & 1.9 & 0.5 & 1.3 & 0.3 & 0.5 & 0.3 & 1.3 & 0.4 & 1.0 & 0.2 \\
\hline Dysthymia & 1.5 & 0.5 & 4.8 & 1.1 & 3.2 & 0.6 & 1.5 & 0.5 & 4.2 & I.I & 2.9 & 0.6 \\
\hline Any affective disorder & 4.9 & 0.7 & 10.7 & 1.2 & 7.9 & 0.8 & 4.2 & 0.6 & 8.6 & I.I & 6.5 & 0.7 \\
\hline \multicolumn{13}{|l|}{ Anxiety disorders } \\
\hline Panic disorder & 0.4 & 0.2 & 1.0 & 0.4 & 0.7 & 0.3 & 0.3 & 0.2 & 0.8 & 0.4 & 0.6 & 0.3 \\
\hline Agoraphobia without panic & 1.5 & 0.5 & 8.5 & $\mathrm{I} .4$ & 5.1 & 0.8 & 1.2 & 0.5 & 7.3 & 1.3 & 4.4 & 0.7 \\
\hline Generalised anxiety disorder & 0.4 & 0.2 & 2.0 & 0.5 & 1.2 & 0.3 & 0.3 & 0.2 & 1.5 & 0.4 & 0.9 & 0.2 \\
\hline Social phobia & 2.4 & 1.0 & 8.8 & 1.9 & 5.9 & 1.4 & 0.8 & 0.1 & 7.7 & 1.5 & 4.5 & 0.9 \\
\hline Simple phobia & 3.1 & 1.3 & 8.8 & $\mathrm{I} .4$ & 6.1 & 1.3 & 2.7 & 1.4 & 7.4 & I.I & 5.2 & 1.2 \\
\hline Obsessive-compulsive disorder & 0.7 & 0.4 & 1.6 & 0.8 & 1.2 & 0.6 & 0.7 & 0.4 & 1.6 & 0.8 & 1.2 & 0.6 \\
\hline Post-traumatic stress disorder & 0.7 & 0.4 & 3.1 & 0.6 & 1.9 & 0.3 & 0.6 & 0.3 & 2.8 & 0.6 & 1.7 & 0.3 \\
\hline Any anxiety disorder & 2.6 & 0.7 & 12.8 & 1.3 & 7.9 & 0.8 & 2.2 & 0.6 & 11.0 & 1.2 & 6.8 & 0.7 \\
\hline \multicolumn{13}{|l|}{ Substance use disorders } \\
\hline Alcohol abuse & 3.5 & 0.7 & 0.7 & 0.3 & 2.0 & 0.3 & 2.6 & 0.6 & 0.6 & 0.3 & 1.6 & 0.3 \\
\hline Alcohol dependence & 7.6 & I.I & 1.4 & 0.5 & 4.3 & 0.6 & 6.5 & 1.1 & 1.3 & 0.5 & 3.8 & 0.6 \\
\hline Drug abuse & 0.5 & 0.3 & 0.1 & 0.1 & 0.3 & 0.2 & 0.0 & 0.0 & 0.0 & 0.0 & 0.0 & 0.0 \\
\hline Drug dependence & 1.0 & 0.5 & 1.7 & 0.6 & 1.3 & 0.4 & 0.9 & 0.5 & 1.5 & 0.5 & 1.2 & 0.4 \\
\hline Nicotine dependence & 2.8 & 0.7 & 3.0 & 0.8 & 2.9 & 0.5 & 2.7 & 0.7 & 2.8 & 0.8 & 2.7 & 0.5 \\
\hline Any alcohol or drug use disorder & 12.0 & 1.3 & 3.5 & 0.6 & 7.6 & 0.7 & 9.6 & 1.3 & 3.2 & 0.6 & 6.3 & 0.7 \\
\hline Any substance use disorder & 13.8 & 1.5 & 6.2 & 1.1 & 9.8 & 1.0 & 11.5 & 1.5 & 5.7 & I.I & 8.5 & 1.0 \\
\hline \multicolumn{13}{|l|}{ Other disorders } \\
\hline Non-affective psychosis ${ }^{2}$ & 0.2 & 0.1 & 0.8 & 0.2 & 0.5 & 0.1 & 0.2 & 0.1 & 0.8 & 0.2 & 0.5 & 0.1 \\
\hline Somatisation disorder & 1.8 & 0.6 & 3.8 & 0.7 & 2.8 & 0.5 & 1.7 & 0.6 & 3.1 & 0.7 & 2.4 & 0.5 \\
\hline Eating disorder & 0.0 & 0.0 & 1.8 & 0.9 & 1.0 & 0.5 & 0.0 & 0.0 & 0.8 & 0.8 & 0.4 & 0.4 \\
\hline Antisocial personality & 1.5 & 0.4 & 0.5 & 0.3 & 1.0 & 0.2 & 1.4 & 0.4 & 0.5 & 0.3 & 0.9 & 0.2 \\
\hline Any CPPS disorder ${ }^{3}$ & 17.9 & $\mathrm{I} .7$ & 21.3 & 1.8 & 19.7 & 1.4 & 14.9 & 1.7 & 18.3 & 1.6 & 16.7 & 1.2 \\
\hline
\end{tabular}

CPPS, Chile Psychiatric Prevalence Study.

I. 'Any anxiety disorder' does not include social phobia, simple phobia or obsessive-compulsive disorder.

2. 'Non-affective psychosis' includes schizophrenia, schizophreniform disorder, schizoaffective disorder, delusional disorder and atypical psychosis.

3. 'Any CPSS disorder' does not include eating disorders, social phobia, simple phobia, obsessive-compulsive disorder, nicotine dependence or cognitive disorder.

were at increased risk of anxiety disorders. For 1-month prevalence, those aged 45-54 years were at increased risk of affective disorders, and those aged 35-54 years of anxiety disorders. For each of the prevalence periods, those under the age of 54 years were at increased risk of antisocial personality disorder, and an increased risk of any disorder was found among those 25-54 years old.

An inverse relationship between educational attainment and overall rates of disorder was not found. Antisocial personality disorder, however, was less prevalent among those without education. An inverse relationship with 6-month prevalence of anxiety disorders and any diagnosis was noted for income, and only for any diagnosis for 1-month prevalence.

Those who were separated, had had their marriages annulled or were never married had the highest rate of affective disorders. For the anxiety disorders, those who were separated or whose marriage had been annulled had significantly higher prevalence rates than respondents who were married, for both 6-month and 1-month prevalence periods. Antisocial personality disorder was more common among those in a common-law relationship.

\section{Comorbidity}

Only about a quarter of individuals with a psychiatric disorder had a comorbid disorder (Table 4). Of those with a disorder, two disorders were present in $14.4 \%$ for 6-month prevalence, and $13.9 \%$ for 1 month prevalence. Three or more disorders were found in less than $11.6 \%$ of those with a disorder. Comorbidity for three or more disorders was significantly higher among women and those under the age of 64 years. Having only basic education was associated with increased comorbidity for 6-month prevalence. For 6-month prevalence, those whose marital status 
Table 2 Socio-demographic correlates of 6-month prevalence rates of mental disorders

\begin{tabular}{|c|c|c|c|c|c|c|c|c|c|c|c|c|}
\hline & \multicolumn{2}{|c|}{ Affective disorder } & \multicolumn{2}{|c|}{ Anxiety disorder } & \multicolumn{2}{|c|}{ Substance disorder } & \multicolumn{2}{|c|}{ Antisocial personality } & \multicolumn{2}{|c|}{ Any diagnosis } & \multicolumn{2}{|c|}{ Three or more disorders } \\
\hline & OR & $(95 \% \mathrm{Cl})$ & OR & $(95 \% \mathrm{Cl})$ & OR & $(95 \% \mathrm{Cl})$ & OR & $(95 \% \mathrm{Cl})$ & OR & $(95 \% \mathrm{Cl})$ & OR & $(95 \% \mathrm{Cl})$ \\
\hline \multicolumn{13}{|l|}{ Gender } \\
\hline Male & 1.00 & & 1.00 & & 1.00 & & 1.00 & & 1.00 & & 1.00 & \\
\hline Female & $2.31^{*}$ & $(1.66-3.23)$ & $5.40^{*}$ & $(2.99-9.76)$ & $0.42^{*}$ & $(0.27-0.63)$ & 0.36 & $(0.11-1.17)$ & 1.25 & $(0.94-1.66)$ & 4.98* & $(2.52-9.87)$ \\
\hline \multicolumn{13}{|l|}{ Age (years) } \\
\hline $15-24$ & 2.36 & $(0.96-5.79)$ & 1.80 & $(0.75-4.32)$ & 1.13 & $(0.34-3.77)$ & $50.86 *$ & (17.39-148.74) & 1.74 & $(0.89-3.38)$ & $29.19 *$ & (8.7I-97.82) \\
\hline $25-34$ & 2.48 & $(1.00-6.15)$ & 2.19 & $(0.97-4.89)$ & 1.53 & $(0.50-4.70)$ & $84.86 *$ & $(43.56-165.31)$ & $1.89 *$ & $(1.02-3.50)$ & $33.74 *$ & $(10.85-104.87)$ \\
\hline $35-44$ & $2.89 *$ & $(1.11-7.52)$ & $2.71 *$ & $(1.2 I-6.05)$ & 1.52 & $(0.55-4.18)$ & $24.88^{*}$ & $(4.09-151.51)$ & $2.10^{*}$ & $(1.08-4.11)$ & $69.46 *$ & (19.77-243.96) \\
\hline $45-54$ & $2.98 *$ & $(1.17-7.58)$ & $2.58^{*}$ & $(1.24-5.35)$ & 1.68 & $(0.48-5.79)$ & $40.06 *$ & $(13.52-118.63)$ & $2.69 *$ & $(1.25-5.78)$ & $50.49 *$ & $(13.93-183.04)$ \\
\hline $55-64$ & 2.44 & $(0.96-6.22)$ & $2.49 *$ & $(1.09-5.66)$ & 1.20 & $(0.36-4.03)$ & 1.00 & $(0.92-1.08)$ & 1.70 & $(0.87-3.32)$ & $46.44 *$ & $(10.52-204.93)$ \\
\hline$\geqslant 65$ & 1.00 & & 1.00 & & 1.00 & & 1.00 & & 1.00 & & 1.00 & \\
\hline \multicolumn{13}{|l|}{ Level of education } \\
\hline No education & 1.00 & & 1.00 & & 1.00 & & 1.00 & & 1.00 & & 1.00 & \\
\hline Basic & 3.17 & $(0.7 I-14.24)$ & 1.44 & $(0.41-5.14)$ & 0.84 & $(0.18-3.88)$ & $29.45^{*}$ & (II.48-75.53) & 1.15 & $(0.4 \mathrm{I}-3.24)$ & 6.22 & $(0.68-57.24)$ \\
\hline Medium & $4.91 *$ & $(1.17-20.59)$ & 1.36 & $(0.40-4.63)$ & 0.77 & $(0.18-3.23)$ & $22.50 *$ & (II.83-42.83) & 1.12 & $(0.45-2.80)$ & $9.16 *$ & $(1.20-70.09)$ \\
\hline High & 2.51 & $(0.57-11.02)$ & 0.74 & $(0.22-2.55)$ & 0.48 & $(0.10-2.24)$ & $11.78 *$ & $(2.82-49.27)$ & 0.52 & $(0.19-1.38)$ & 3.29 & $(0.36-29.85)$ \\
\hline \multicolumn{13}{|l|}{ Income (US\$) } \\
\hline $100-400$ & 1.00 & & 1.00 & & 1.00 & & 1.00 & & 1.00 & & 1.00 & \\
\hline $40 \mathrm{I}-800$ & 1.32 & $(0.88-1.98)$ & 0.79 & $(0.49-1.27)$ & 1.23 & $(0.69-2.22)$ & 1.49 & $(0.17-1.42)$ & 1.18 & $(0.85-1.64)$ & 0.62 & $(0.26-I .5 I)$ \\
\hline $80 I-1500$ & $0.57^{*}$ & $(0.36-0.90)$ & $0.38^{*}$ & $(0.20-0.74)$ & 1.46 & $(0.92-2.30)$ & 1.47 & $(0.22-9.63)$ & $0.66 *$ & $(0.49-0.88)$ & 1.12 & $(0.47-2.68)$ \\
\hline$\geqslant|50|$ & 0.82 & $(0.45-1.48)$ & $0.38^{*}$ & $(0.17-0.86)$ & $1.64^{*}$ & $(1.07-2.53)$ & 1.44 & $(0.21-9.77)$ & $0.59 *$ & $(0.40-0.87)$ & 1.07 & $(0.49-2.34)$ \\
\hline \multicolumn{13}{|l|}{ Marital status } \\
\hline Married & 1.00 & & 1.00 & & 1.00 & & 1.00 & & 1.00 & & 1.00 & \\
\hline Widowed & 1.33 & $(0.62-2.85)$ & 1.04 & $(0.47-2.30)$ & 0.85 & $(0.32-2.25)$ & $0.03 *$ & $(0.02-0.07)$ & 0.83 & $(0.49-1.40)$ & 1.38 & $(0.34-5.52)$ \\
\hline $\begin{array}{l}\text { Separated/ } \\
\text { annulled }\end{array}$ & $2.7 I^{*}$ & $(1.52-4.84)$ & $2.61 *$ & $(1.28-5.32)$ & 1.52 & $(0.82-2.84)$ & 2.39 & $(0.27-21.49)$ & $1.91 *$ & $(1.09-3.34)$ & $5.52 *$ & $(1.94-15.73)$ \\
\hline Never married & $1.87^{*}$ & $(1.31-2.68)$ & 0.85 & $(0.51-1.42)$ & 1.07 & $(0.55-2.10)$ & 1.01 & $(0.28-3.63)$ & 1.00 & $(0.70-I .4 I)$ & $2.84 *$ & $(1.05-7.65)$ \\
\hline Common law & 1.35 & $(0.63-2.88)$ & 1.20 & $(0.57-2.51)$ & 1.25 & $(0.63-2.48)$ & $7.04 *$ & $(2.06-24.00)$ & 1.20 & $(0.69-2.08)$ & $3.58^{*}$ & $(1.10-11.65)$ \\
\hline
\end{tabular}

was 'separated or annulled' and those who had never been married had more comorbidity than those who were married, whereas those who were widowed had lower rates of comorbidity. For 1-month prevalence, those living in common-law relationships had more comorbidity than those who were married. All those diagnosed with PTSD also had generalised anxiety disorder, agoraphobia or panic disorder.

\section{Service use}

About $30 \%$ of people who had a single psychiatric disorder had sought some type of mental health care in this study. Of those who had three or more disorders, the majority had sought mental health treatment. Less than $12 \%$ of individuals with any psychiatric disorder received treatment from a specialist. A sizeable number of individuals without any psychiatric disorder according to the CPPS also had sought services for mental health care (Table 5).

\section{DISCUSSION}

A fifth of the Chilean population surveyed was found to have had a psychiatric disorder in the preceding 6 months and nearly $17 \%$ met criteria for a current psychiatric disorder. The five most common disorders were simple phobia, social phobia, agoraphobia, major depressive disorder and alcohol dependence. Among men, alcohol misuse and dependence was the most common disorder, whereas anxiety disorders were the most prevalent in women. Less than a third were found to have a comorbid disorder. Over $60 \%$ of those with a current psychiatric diagnosis failed to obtain any form of medical attention for their disorder, and less than $12 \%$ received specialist care.

\section{Limitations}

The results of this study should be evaluated in the context of its limitations. The CIDI, although widely used, is administered by lay interviewers and does not allow clinical interpretation or probing beyond its structured format. The sample size, although larger than in most Latin American studies, might have been insufficient to yield the power needed to examine risk factors of low-prevalence disorders. This may in part account for the limited findings on the role of socio-economic status variables. Interviews were not conducted simultaneously in the four catchment areas, but over 7 years, owing to the major social 
Table 3 Socio-demographic correlates of I-month prevalence rates of mental disorders

\begin{tabular}{|c|c|c|c|c|c|c|c|c|c|c|c|c|}
\hline & \multicolumn{2}{|c|}{ Affective disorder } & \multicolumn{2}{|c|}{ Anxiety disorder } & \multicolumn{2}{|c|}{ Substance disorder } & \multicolumn{2}{|c|}{ Antisocial personality } & \multicolumn{2}{|c|}{ Any diagnosis } & \multicolumn{2}{|c|}{ Three or more disorders } \\
\hline & OR & $(95 \% \mathrm{Cl})$ & OR & $(95 \% \mathrm{Cl})$ & OR & $(95 \% \mathrm{Cl})$ & OR & $(95 \% \mathrm{Cl})$ & OR & $(95 \% \mathrm{Cl})$ & OR & $(95 \% \mathrm{Cl})$ \\
\hline \multicolumn{13}{|l|}{ Gender } \\
\hline Male & 1.00 & & 1.00 & & 1.00 & & 1.00 & & 1.00 & & 1.00 & \\
\hline Female & $2.14 *$ & $(1.49-3.07)$ & $5.42 *$ & $(2.82-10.40)$ & $0.46^{*}$ & $(0.29-0.74)$ & 0.39 & $(0.12-1.28)$ & 1.28 & $(0.91-1.8 I)$ & $4.45^{*}$ & $(2.05-9.63)$ \\
\hline \multicolumn{13}{|l|}{ Age (years) } \\
\hline $15-24$ & 1.87 & $(0.73-4.79)$ & 1.84 & $(0.65-5.22)$ & 1.46 & $(0.5 I-4.24)$ & $51.84 *$ & $(17.72-151.62)$ & $2.02 *$ & $(1.13-3.59)$ & $25.14 *$ & $(7.00-90.25)$ \\
\hline $25-34$ & 1.80 & $(0.72-4.54)$ & 2.00 & $(0.79-5.04)$ & 2.21 & $(0.80-6.07)$ & $77.32 *$ & (38.38-155.75) & $2.14 *$ & $(1.17-3.92)$ & $24.06 *$ & $(7.58-76.33)$ \\
\hline $35-44$ & 2.36 & $(0.87-6.36)$ & $2.51 *$ & $(1.02-6.16)$ & 2.11 & $(0.75-5.93)$ & $25.36^{*}$ & $(4.16-154.48)$ & $2.39 *$ & $(1.25-4.58)$ & $51.35 *$ & $(12.92-204.10)$ \\
\hline $45-54$ & $3.0 I^{*}$ & $(1.15-7.90)$ & $2.43^{*}$ & $(1.10-5.37)$ & 2.28 & $(0.7 I-7.35)$ & $40.83 *$ & $(13.78-120.93)$ & $3.35 *$ & $(1.97-5.70)$ & $49.80 *$ & $(18.63-181.90)$ \\
\hline $55-64$ & 2.25 & $(0.83-6.1)$ & 2.33 & $(0.90-6.06)$ & 1.82 & $(0.52-6.33)$ & 1.00 & $(0.93-1.08)$ & $2.06 *$ & $(1.20-3.52)$ & $42.36^{*}$ & $(8.62-208.08)$ \\
\hline$\geqslant 65$ & 1.00 & & 1.00 & & 1.00 & & 1.00 & & 1.00 & & 1.00 & \\
\hline \multicolumn{13}{|l|}{ Level of education } \\
\hline No education & 1.00 & & 1.00 & & 1.00 & & 1.00 & & 1.00 & & 1.00 & \\
\hline Basic & $6.03 *$ & $(1.05-34.6)$ & 1.77 & $(0.43-7.31)$ & 0.65 & $(0.13-3.23)$ & $31.01 *$ & (12.11-79.45) & 1.09 & $(0.33-3.63)$ & 6.15 & $(0.66-56.87)$ \\
\hline Medium & $8.22 *$ & $(1.66-40.67)$ & 1.56 & $(0.40-6.0 I)$ & 0.66 & $(0.16-2.63)$ & $21.61 *$ & (II.I5-4I.88) & 1.05 & $(0.37-3.00)$ & 7.24 & $(0.94-55.99)$ \\
\hline High & 3.68 & $(0.68-19.87)$ & 0.88 & $(0.23-3.34)$ & 0.42 & $(0.09-1.97)$ & $12.40 *$ & $(2.98-51.60)$ & 0.49 & $(0.17-1.42)$ & 2.09 & $(0.23-19.19)$ \\
\hline \multicolumn{13}{|l|}{ Income (US\$) } \\
\hline $100-400$ & 1.00 & & 1.00 & & 1.00 & & 1.00 & & 1.00 & & 1.00 & \\
\hline $40 \mathrm{I}-800$ & 0.92 & $(0.60-I .4 I)$ & 0.84 & $(0.46-1.54)$ & 1.20 & $(0.60-2.37)$ & 0.49 & $(0.17-1.42)$ & 1.05 & $(0.79-1.40)$ & 0.49 & $(0.18-1.35)$ \\
\hline $80 I-I 500$ & $0.49 *$ & $(0.29-0.8 I)$ & $0.42 *$ & $(0.20-0.88)$ & 1.39 & $(0.85-2.29)$ & 1.47 & $(0.22-9.63)$ & $0.60 *$ & $(0.43-0.84)$ & $\mathrm{I} .24$ & $(0.5 I-3.05)$ \\
\hline$\geqslant 1501$ & 0.54 & $(0.29-1.02)$ & 0.40 & $(0.14-1.13)$ & 1.67 & $(0.99-2.80)$ & $\mathrm{I} .44$ & $(0.21-9.77)$ & $0.63 *$ & $(0.42-0.94)$ & $0.26 *$ & $(0.09-0.77)$ \\
\hline \multicolumn{13}{|l|}{ Marital status } \\
\hline Married & 1.00 & & 1.00 & & 1.00 & & 1.00 & & 1.00 & & 1.00 & \\
\hline Widowed & $\mathrm{I} .4 \mathrm{I}$ & $(0.65-3.10)$ & 0.94 & $(0.38-2.31)$ & 0.98 & $(0.36-2.66)$ & $0.03 *$ & $(0.02-0.07)$ & 0.80 & $(0.45-1.43)$ & 0.45 & $(0.36-5.84)$ \\
\hline $\begin{array}{l}\text { Separated/ } \\
\text { annulled }\end{array}$ & $1.64 *$ & $(1.06-2.54)$ & $1.96 *$ & $(1.06-3.62)$ & 1.58 & $(0.85-2.95)$ & 2.39 & $(0.27-21.49)$ & 1.58 & $(0.95-2.63)$ & 2.97 & $(0.78-11.26)$ \\
\hline Never married & $1.75^{*}$ & $(1.24-2.48)$ & 0.87 & $(0.48-1.57)$ & I.II & $(0.58-2.13)$ & 0.80 & $(0.20-3.17)$ & 0.97 & $(0.70-1.35)$ & 2.16 & $(0.69-6.76)$ \\
\hline Common law & 1.59 & $(0.77-3.29)$ & 1.06 & $(0.53-2.1 \mathrm{I})$ & 1.29 & $(0.64-2.59)$ & $7.04 *$ & $(2.06-24.00)$ & 1.17 & $(0.70-1.96)$ & $3.63 *$ & $(1.08-12.28)$ \\
\hline
\end{tabular}

$* P<0.05$ (two-tailed).

Table 4 Comorbidity in 6-month and I-month prevalent disorders

\begin{tabular}{|c|c|c|c|c|}
\hline \multirow[t]{2}{*}{ Number of lifetime disorders } & \multicolumn{2}{|c|}{$\begin{array}{l}\text { Proportion with 6-month } \\
\text { diagnosis }\end{array}$} & \multicolumn{2}{|c|}{$\begin{array}{c}\text { Proportion with I-month } \\
\text { diagnosis }\end{array}$} \\
\hline & $\%$ & s.e. & $\%$ & s.e. \\
\hline I & 74.0 & 3.0 & 75.1 & 3.2 \\
\hline 2 & 14.4 & 1.6 & 13.9 & 2.3 \\
\hline 3 or more & 11.6 & 2.0 & 11.0 & 1.9 \\
\hline
\end{tabular}

and political changes that occurred during this time; this is a reflection of the funding difficulties of conducting research in a developing country.

\section{Comparison with other Latin American studies}

Overall, some consistency is found in the prevalence rates reported by studies conducted in Spanish-speaking Latin America (Table 6). For major depression, Colombia (Torres de Galvis \& Montoya, 1997) and Puerto Rico (Canino et al, 1987) appeared to have considerably lower prevalence rates; the Puerto Rico rates could be explained by the country's geographical distance from South America and because the study was conducted a decade earlier using the DIS rather than the CIDI. The reason for the virtual absence of panic disorder in Colombia in contrast to the other countries in the region may be methodological. The low rate of dysthymia and generalised anxiety disorder in Mexico (Caraveo-Anduaga et al, 1996) compared with Chile is difficult to explain, especially since the Chilean rate is closer to other international studies (Kessler et al, 1994). The rate of comorbidity in Chile is low compared with studies in the USA (Kessler et al, 1994); however, it is unknown if this finding is generalisable to other Spanishspeaking countries in Latin America.

Perhaps the most intriguing comparisons with the current study come from another Chilean survey, by Araya et al (2001), restricted to the city of Santiago. This study used the Clinical Interview Schedule - Revised (CIS-R; Lewis et al, 1992) and obtained a sample representative 


\begin{tabular}{|c|c|c|c|c|c|c|c|c|}
\hline & \multicolumn{2}{|c|}{ No diagnosis } & \multicolumn{2}{|c|}{ Any disorder } & \multicolumn{2}{|c|}{ One disorder } & \multicolumn{2}{|c|}{ Three or more disorders } \\
\hline & $\%$ & s.e. & $\%$ & s.e. & $\%$ & s.e. & $\%$ & s.e. \\
\hline \multicolumn{9}{|l|}{ Six-month prevalence } \\
\hline Any mental health service & 15.2 & 1.0 & 39.0 & 3.9 & 30.9 & 2.9 & 77.0 & 5.5 \\
\hline Non-specialist mental health service & I3.1 & 1.0 & 35.0 & 3.1 & 28.9 & 2.7 & 61.6 & 7.6 \\
\hline Specialist mental health service & 4.0 & 0.8 & 11.8 & 2.1 & 8.6 & 2.0 & 27.2 & 10.3 \\
\hline Substance misuse service & 0.05 & 0.04 & 0.8 & 0.4 & 0.2 & 0.2 & 2.9 & 1.9 \\
\hline \multicolumn{9}{|l|}{ One-month prevalence } \\
\hline Any mental health service & 15.9 & I.I & 39.8 & 4.4 & 31.8 & 3.3 & 77.0 & 6.8 \\
\hline Non-specialist mental health service & 13.9 & I.I & 35.4 & 3.5 & 29.8 & 3.2 & 59.0 & 8.4 \\
\hline Specialist mental health service & 4.4 & 0.8 & 11.5 & 2.6 & 7.8 & 2.3 & 31.0 & 12.1 \\
\hline Substance misuse service & 0.08 & 0.05 & 0.8 & 0.4 & 0.1 & 0.1 & 3.7 & 2.3 \\
\hline
\end{tabular}

Table 6 Comparison of prevalence rates in Spanish-speaking Latin American countries

\begin{tabular}{|c|c|c|c|c|c|c|c|}
\hline & CPPS' & Santiago ${ }^{2}$ & Colombia $^{3}$ & $\operatorname{Lima}^{4}$ & Mexico $^{5}$ & Mexico City ${ }^{6}$ & Puerto Rico ${ }^{7}$ \\
\hline Major depressive disorder & 4.7 & 5.5 & 1.9 & 5.2 & 3.2 & 4.4 & 2.3 \\
\hline Dysthymia & 3.2 & & & 3.3 & & 0.3 & \\
\hline Manic episode & 1.3 & & & 0.1 & 0.5 & 0.7 & 0.3 \\
\hline Schizophrenia & 0.5 & & 0.6 & 0.6 & 0.7 & & 1.7 \\
\hline Generalised anxiety disorder & 1.2 & 5.1 & 1.3 & & & 0.6 & \\
\hline Panic disorder & 0.7 & 1.3 & 0.1 & 1.6 & & 1.6 & I.I \\
\hline Alcohol misuse/dependence & 6.3 & & 4.7 & 6.9 & & 5.6 & 6.1 \\
\hline
\end{tabular}

CIDI, Composite International Diagnostic Interview; CIS-R, Clinical Interview Schedule - Revised; CPPS, Chile Psychiatric Prevalence Study; DIS, Diagnostic Interview Schedule;

PSE, Present State Examination.

I. Age range, I5+ years; diagnostic instrument, CIDI; prevalence period, 6 months.

2. Age range, I6-64 years; diagnostic instrument, CIS-R; prevalence period, current (Araya et al, 200I).

3. Age range, I2+ years; diagnostic instrument, CIDI; prevalence period, I year (Torres de Galvis \& Montoya, 1997).

4. Age range, 18+ years; diagnostic instrument, DIS; prevalence period, 6 months (Hayashi et al, 1985).

5. Age range, 18-64 years; diagnostic instrument, PSE; prevalence period, current (Caraveo-Anduaga, 1995).

6. Age range, 18-64 years; diagnostic instrument, CIDI; prevalence period, I year (Caraveo-Anduaga et al, 1996).

7. Age range, 18+ years; diagnostic instrument, DIS; prevalence period, 6 months (Canino et al, 1987).

of the entire city of Santiago diagnosed using ICD-10 criteria (World Health Organization, 1992). The CPPS included Santiago as one of its sites, with a sampling of selected barrios, in which prevalence rates were similar to the overall national rates. These two studies have disparate prevalence rates. The most striking difference is for major depression and generalised anxiety disorders: the Santiago study has current prevalence rates of $5.5 \%$ and $5.1 \%$, whereas the CPPS has 1 -month prevalence rates of $3.4 \%$ and $0.9 \%$, respectively. The differences are most probably methodological: first, the DSM and ICD systems do not necessarily yield the same prevalence rates (Andrews \& Slade, 2002); second, the CIS-R is based on response to a set of symptom scales from which diagnosis is obtained based on established cut-off values, (a 'bottom-up' approach), whereas the CIDI is based on responses to specific diagnostic criteria that lead the interviewer to follow an algorithm that determines the presence of a disorder (a 'top-down' approach). The contrasting results in these two studies merit further investigation, as such widely different findings may lead policy planners to distrust the results of both studies.

The use of current prevalence $v$. 6month prevalence rates also raises the issue of which rate is the most meaningful. Studies limited to 1-month prevalence data fail to ascertain a fully representative group of individuals with mental illness, in particular those at risk of relapse or those who have successfully responded to treatment but are still in need of services. One-month data in service planning would therefore underestimate future mental health needs.

Psychiatric epidemiological studies in Latin America need to evolve further. More data are needed from other countries in the region to facilitate planning. Measures of disability, service use and comorbidity need to be incorporated into future studies. In addition, longitudinal studies exploring remission and the risk of relapse are needed for this region. However, the research conducted so far supports the epidemiological call to action to address the growing burden of mental illness in Latin America.

\section{ACKNOWLEDGEMENTS}

The authors thank the Pan American Health Organization and the World Health Organization for their technical and financial support. We also 
acknowledge the financial support of FONDECYT (no. 90-229, 920233, 1971315, 1990325) and Dirección de Investigación de la Universidad de Concepción (no. 201.087.027-1.0).

\section{REFERENCES}

American Psychiatric Association (1987) Diagnostic and Statistical Manual of Mental Disorders (3rd edn, revised) (DSM-III-R). Washington, DC: APA

Andrews, G. \& Slade, T. (2002) The classification of anxiety disorders in ICD-10 and DSM-IV: a concordance analysis. Psychopathology, 35, 100-106.

Araya, R., Rojas, G., Fritsch, R., et al (200I) Common mental disorders in Santiago, Chile: prevalence and socio-demographic correlations. British Journal of Psychiatry, 178, 228-233.

Canino, G. J., Bird, H. R., Shrout, P. E., et al (1987)

The prevalence of specific psychiatric disorders in Puerto Rico. Archives of General Psychiatry, 44, 727-735.

Caraveo-Anduaga, J. (1995) Epidemiologia de la Morbilidad Psiquiátrica en la Ciudad de México. Mexico City: Instituto Mexicano de Psiquiatria.

Caraveo-Anduaga, J., Medina-Mora, M. E., Rascón, M. L., et al (1996) Lá prevalencia de los trastornos psiquiátricos en la población urbana adulta en México. Salud Mental, 19, |4-2|.

Hayashi, S., Perales, A., Sogi, C., et al (1985) Prevalencia de vida de trastornos mentales en Independencia (Lima, Peru). Anales de Salud Mental, I, 206-222.

Kessler, R. C., McGonagle, K. A., Zhao, S., et al (1994) Lifetime and I2-month prevalence of DSM-III-R psychiatric disorders in the United States. Archives of General Psychiatry, 5I, 8-19.

Kish, L. (1965) Survey Sampling. New York: John Wiley.

Larraya, F. P., Casullo, M. M. \& Viola, F. P. (1982)

Prevalencia de la Patologia Mental en la Megalopolis de Buenos Aires. Buenos Aires: Consejo Nacional de Investigaciones Cientificas y Tecnicas.

Larrobla, C. \& Botega, N. J. (200I) Restructuring mental health: a South American survey. Social Psychiatry and Psychiatric Epidemiology, 36, 256-259.

Levav, I., Lima, B. R., Somoza Lennon, M., et al (1989) Epidemiologic bases for action. Boletín de la Oficina Sanitaria Panamericana, 107, 196-219.

Lewis, G., Pelosi, A. J., Araya, R., et al (1992)

Measuring psychiatric disorder in the community: a standardized assessment for use by lay interviewers. Psychological Medicine, 22, 465-486.

Murray, C. J. L. \& Lopez, A. D. (eds) (1996) The Global Burden of Disease: A Comprehensive Assessment of Mortality and Disability from Diseases, Injuries, and Risk Factors in 1990 and Projected to 2020. Cambridge, MA: Harvard University Press.

\section{CLINICAL IMPLICATIONS}

More than two-thirds of people with a single psychiatric disorder failed to receive any treatment.

- In men, alcohol misuse and dependence were found to be the most common disorder.

- The growing burden of mental illness in Latin America must be addressed.

\section{LIMITATIONS}

- The four catchment areas were not studied simultaneously but over a 7-year period.

- The sample size might have been insufficient for low-prevalence disorders.

- The diagnostic interview was administered by lay investigators.

BENJAMIN VICENTE, MD, PhD, Department of Psychiatry and Mental Health, University of Concepción Concepción, Chile; ROBERT KOHN, MD, Department of Psychiatry and Human Behavior, Brown University, Providence, Rhode Island, USA; PEDRO RIOSECO, MD, SANDRA SALDIVIA, PhD, University of Concepción, Concepción, Chile; CHRISTINE BAKER, MPH, Department of Psychiatry and Human Behavior, Brown University, Providence, Rhode Island, USA; SILVERIO TORRES, University of Concepción, Concepción, Chile

Correspondence: Professor B.Vicente, Universidad de Concepción, Departamento de Psiquiatría y Salud Mental, Casilla60-C,Concepción, Chile. Tel/fax: +56 4 I3l 2799; e-mail: bvicent@udec.cl

(First received 28 May 2003, final revision 12 September 2003, accepted 2 October 2003)

Rioseco, P., Vicente, B., Uribe, M., et al (1992) EI DISIII-R una validación en Chile. Revista de Psiquiatría, 9 1034-1038

Robins, L. N., Helzer, J. E., Croughan, J., et al (198I) National Institute of Mental Health diagnostic interview schedule: its history, characteristics, and validity. Archives of General Psychiatry, 38, 38I-389.

Robins, L. N., Wing, J., Wittchen, H. U., et al (1988) The Composite International Diagnostic Interview: an epidemiologic instrument suitable for use in conjunction with different diagnostic systems and in different cultures. Archives of General Psychiatry, 45, 1069-1077.

Sartorius, N. \& Kuyken, W. (1994) Translation of health status instruments. In Quality of Life Assessment in Health Care Settings, vol. I (eds J. Orley \& W. Kuyken), pp. 3-18. Berlin: Springer.
Shah, B. V., Barnwell, B. G. \& Bieler, G. S. (1997) SUDAAN User's Manual, Release 7.5. Research Triangle Park, NC: Research Triangle Institute.

Torres de Galvis, Y. \& Montoya, L. D. (1997) Segundo Estudio Nacional de Salud Mental y Consumo de Sustancias Psicoactivas Colombia 1997. Colombia: Ministry of Health

Vielma, M., Vicente, B., Rioseco, P., et al (1992) Validación en Chile de la entrevista diagnóstica estandarizada para estudios epidemiológicos CIDI. Revista de Psiquiatria 9, 1039-1049.

World Bank (2002) World Development Indicators 2002 Washington, DC: World Bank.

World Health Organization (1992) International Classification of Diseases and Related Disorders (ICD-10). Geneva: WHO. 